\title{
Hydrodynamic Mixed Convection in a Lid-Driven Hexagonal Cavity with Corner Heater
}

\author{
M. Jahirul Haque Munshi1 ${ }^{*}$, Golam Mostafa1, A. B. S. Manik Munsi², Md. Waliullah' \\ ${ }^{1}$ Department of Mathematics, Hamdard University Bangladesh, Munshiganj, Bangladesh \\ ${ }^{2}$ Department of Mathematics, Jagannath University, Dhaka, Bangladesh \\ Email: *jahir.buet.bd@gmail.com, mostafamath02@gmail.com, abs.manik@math.jnu.ac.bd
}

How to cite this paper: Munshi, M.J.H., Mostafa, G., Munsi, A.B.S.M. and Waliullah, Md. (2018) Hydrodynamic Mixed Convection in a Lid-Driven Hexagonal Cavity with Corner Heater. American Journal of Computational Mathematics, 8, 245-258. https://doi.org/10.4236/ajcm.2018.83020

Received: July 17, 2018

Accepted: September 18, 2018

Published: September 21, 2018

Copyright ( 92018 by authors and Scientific Research Publishing Inc. This work is licensed under the Creative Commons Attribution International License (CC BY 4.0).

http://creativecommons.org/licenses/by/4.0/

\begin{abstract}
Hydrodynamic mixed convection in a lid-driven hexagonal cavity with corner heater is numerically simulated in this paper by employing finite element method. The working fluid is assigned as air with a Prandtl number of 0.71 throughout the simulation. The left and right walls of the hexagonal cavity are kept thermally insulated and the lid moves top to bottom at a constant speed $U_{0}$. The top left and right walls of the enclosure are maintained at cold temperature $T_{c}$. The bottom right wall is considered with a corner heater whereas the bottom remaining part is adiabatic and inside the cavity a square shape heated block $T_{h}$. The focus of the work is to investigate the effect of Hartmann number, Richardson number, Grashof number and Reynolds number on the fluid flow and heat transfer characteristics inside the enclosure. A set of graphical results is presented in terms of streamlines, isotherms, local Nusselt number, velocity profiles, temperature profiles and average Nusselt numbers. The results reveal that heat transfer rate increases with increasing Richardson number and Hartmann number. It is also observed that, Hartmann number is a good control parameter for heat transfer in fluid flow in hexagonal cavity.
\end{abstract}

\section{Keywords}

Mixed Convection, Lid-Driven Hexagonal Cavity, Finite Element Method, Square Block, Corner Heater

\section{Introduction}

Mixed convection in enclosures is encountered in many engineering systems 
such as cooling of electronic components, ventilation in buildings and fluid movement in solar energy collectors, astrophysics, geology, biology and chemical processes, as well as in many engineering applications. Also mixed convection involving the combined effect of forced and natural convection has been the focus of research due to its occurrence in numerous technological, engineering and natural applications such as: cooling of electronic devices, lubrication technologies, drying technologies, and food processing. Al-Amiri et al. [1] numerically investigated steady mixed convection in a square lid-driven cavity under the combined buoyancy effects of thermal and mass diffusion. The results demonstrate the range where high heat and mass transfer rates can be attained for a given Richardson number. Sharif [2] studied numerically laminar mixed convective heat transfer in two-dimensional shallow rectangular driven cavities of aspect ratio 10. The top moving lid of the cavity is at a higher temperature than the bottom wall. The effects of inclination of the cavity on the flow and thermal fields are investigated. The stream line and isotherm plots and the variation of the local and average Nusselt numbers at the hot and cold walls are presented. Chen and Cheng [3] investigated numerically the Periodic behavior of the mixed convective flow in a rectangular cavity with a vibrating lid. The periodic flow patterns and heat transfer characteristics found are discussed with attention being focused on the interaction between the frequency of the lid velocity vibration and the frequency of the natural periodic flow. Khanafer et al. [4] investigated numerically unsteady laminar mixed convection heat transfer in a lid driven cavity. M. A. Teamah et al. [5] have performed a numerical simulation of double-diffusive mixed convective flow in rectangular enclosure with insulated moving lid. Saha et al. [6] have performed the numerical effect of internal heat generation or absorption on Magneto-Hydro Dynamics (MHD) mixed convection flow in a lid driven cavity. Significant reduction in the average Nusselt number was produced as the strength of the applied magnetic field was increased. In addition, heat generation predicated to decrease the average Nusselt number whereas heat absorption increases it. Dawood et al. [7] investigated hydro-magnetic mixed Convection double diffusive in a Lid Driven Square Cavity. Hussein [8] investigated the study of mixed convection in square lid-driven with eccentric circular body. Nasrin [9] carried out an aspect ratio effect of vertical lid-driven chamber having a centered conducting solid on mixed magneto convection. Billah et al. [10] investigated the numerical analysis of fluid flow due to mixed convection in a lid-driven cavity having a heated circular hollow cylinder. Munshi et al. [11] investigated hydrodynamic mixed convection in a lid-driven square cavity including elliptic shape heated block with corner heater. Sivasankaran et al. [12] examined Hydro-magnetic combined convection in a lid-driven cavity with sinusoidal boundary conditions on both sidewalls.

To the best of author's understanding, little attention is given to the problem of MHD mixed convection in a lid-driven cavity with corner heater. There is no previous study on hydrodynamic mixed convection in a lid-driven hexagonal 
cavity with a corner heater and inside the square heated block. Therefore, this problem could be occurred in many engineering applications such as conveyer belt, escalator and lift as elevator, heating and cooling flows in buildings. The objective of the present study is to investigate numerically hydrodynamic mixed convection in a lid-driven hexagonal cavity with a corner heater, also increase of the lid-driven constant velocity to highlight the applicability of the approach. Numerical results are presented via streamlines, isotherms, velocity profiles, dimensionless temperature, local Nusselt number and average Nusselt number to analyze the effect of Richardson number and Hartmann number of the fluid flow and heat transfer.

The paper is arranged in the following manner: in Section 2, we consider a physical configuration of hexagonal cavity; Section 3 presents the mathematical formulation of this model, corresponding boundary conditions and grid generation for that cavity; in Section 4, detailed analysis of different results and corresponding discussions is added; finally, a brief conclusion is given in Section 5.

\section{Physical Configuration}

The considered two-dimensional model in the present study of mixed convection in a hexagonal cavity with internal heated square block is shown in Figure 1. As seen from the schematically view, the left and right walls are lid-driven and move top to bottom with constant velocity. The upper two walls are cold. The left, right and left lower corner walls are adiabatic. A corner heater with temperature is located in the right lower corner. The magnetic field of strength $B_{0}$ is applied parallel to $\boldsymbol{X}$-axis. A square block with temperature $T_{h}$ is located inside the enclosure at middle position.

\section{Mathematical Formulation}

The fluid is considered as incompressible, Newtonian and the flow is assumed to

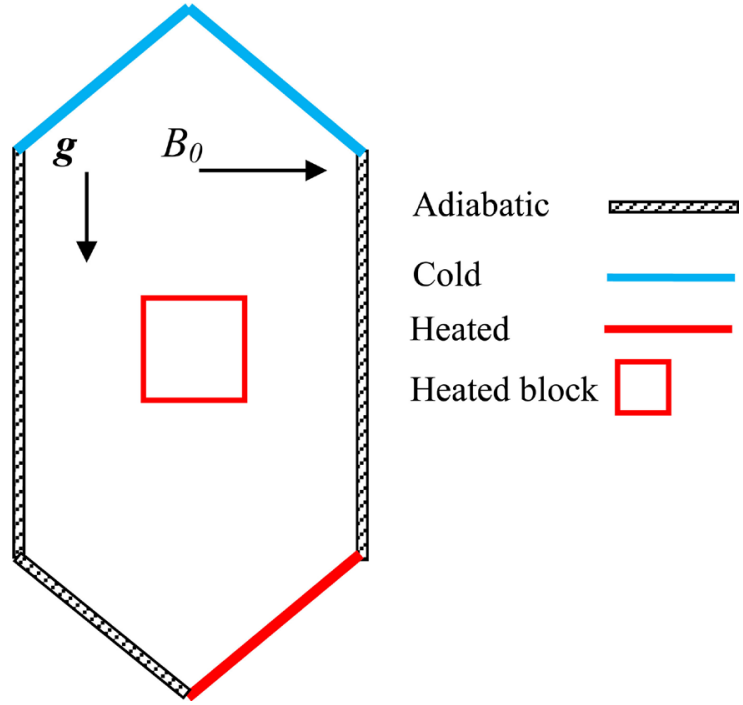

Figure 1. Schematic view of the hexagonal cavity. 
be laminar. Two dimensional, steady equations are written by considering a uniform applied magnetic field. It is assumed that Boussinesq approximation is valid and radiation mode of heat transfer, Joule heating and Hall effects are neglected according to other modes of heat transfer. Thus, using the coordinate system shown in Figure 1, the governing equations for mass, momentum and energy equation can be written in dimensional form as follows:

$$
\begin{gathered}
\frac{\partial u}{\partial x}+\frac{\partial v}{\partial y}=0 \\
u \frac{\partial u}{\partial x}+v \frac{\partial u}{\partial y}=-\frac{1}{\rho} \frac{\partial p}{\partial x}+v\left(\frac{\partial^{2} u}{\partial x^{2}}+\frac{\partial^{2} u}{\partial y^{2}}\right) \\
u \frac{\partial v}{\partial x}+v \frac{\partial v}{\partial y}=-\frac{1}{\rho} \frac{\partial p}{\partial y}+v\left(\frac{\partial^{2} v}{\partial x^{2}}+\frac{\partial^{2} v}{\partial y^{2}}\right)-\frac{\sigma B_{0}^{2}}{\rho} v+g \beta\left(T-T_{c}\right) \\
u \frac{\partial T}{\partial x}+v \frac{\partial T}{\partial y}=\alpha\left(\frac{\partial^{2} T}{\partial x^{2}}+\frac{\partial^{2} T}{\partial y^{2}}\right)
\end{gathered}
$$

\subsection{Boundary Conditions}

The boundary conditions for the present problem are specified as follows:

On the top left and right walls: $u=0, v=0, T=T_{c}$

On the left and right walls: $u=0, v=-v_{0}, \frac{\partial T}{\partial x}=0$

On the lower left wall: $u=0, v=0, \frac{\partial T}{\partial x}=0$

On the lower right wall and square block: $u=0, v=0, T=T_{h}$ where $x$ and $y$ are the distance measured along the horizontal and the vertical directions respectively, $u$ and $v$ are the velocity components in $x$ and $y$ direction respectively, $T$ denotes the temperature, $v$ denotes the kinematic viscosity, $\alpha$ denotes the thermal diffusivity respectively, $p$ is the pressure and $\rho$ is the density.

The governing equations are non-dimensionalized by using the following dimensionless quantities:

$$
X=\frac{x}{L}, Y=\frac{y}{L}, U=\frac{u}{U_{0}}, V=\frac{v}{U_{0}}, P=\frac{p}{\rho U_{0}^{2}}, \theta=\frac{T-T_{c}}{T_{h}-T_{c}}
$$

After substitution of dimensionless variable we get the non-dimensional governing equations which are:

$$
\begin{gathered}
\frac{\partial U}{\partial X}+\frac{\partial V}{\partial Y}=0 \\
U \frac{\partial U}{\partial X}+V \frac{\partial U}{\partial Y}=-\frac{\partial P}{\partial X}+\operatorname{Pr}\left(\frac{\partial^{2} U}{\partial X^{2}}+\frac{\partial^{2} U}{\partial Y^{2}}\right) \\
U \frac{\partial V}{\partial X}+V \frac{\partial V}{\partial Y}=-\frac{\partial P}{\partial Y}+\operatorname{Pr}\left(\frac{\partial^{2} V}{\partial X^{2}}+\frac{\partial^{2} V}{\partial Y^{2}}\right)-H a^{2} \operatorname{Pr} V+\operatorname{RiPr} \theta
\end{gathered}
$$




$$
U \frac{\partial \theta}{\partial X}+V \frac{\partial \theta}{\partial Y}=\frac{1}{\operatorname{RePr}}\left(\frac{\partial^{2} \theta}{\partial X^{2}}+\frac{\partial^{2} \theta}{\partial Y^{2}}\right)
$$

where $U$ and $V$ are the velocity components in $X$ and $Y$ directions respectively, $P$ is the pressure and $\theta$ is the non-dimensional temperature. As we know $R i=G r / R e^{2}$, when $R i \approx 1$ both free and forced convection are equally dominant and the flow regime is designated as mixed convection. If $R i>1$ then free convection is dominant whereas forced convection is dominant when $R i<1$.

\section{The transformed boundary conditions are:}

On the top left and right walls: $\theta=0, U=0, V=0$

On the left and right walls: $U=0, V=-1, \frac{\partial \theta}{\partial X}=0$

On the lower left wall: $U=0, V=0, \frac{\partial \theta}{\partial X}=0$

On the lower right wall and square block: $\theta=1, U=0, V=0$

\subsection{Grid Refinement Check}

We examined five different non-uniform grid systems with the following number of elements within the resolution field. It is observed from Table 1 that grid independence is achieved with 25,482 elements where there is insignificant change in $\mathrm{Nu}$ with further increase of mesh elements. Five different non-uniform grids with the following number of nodes and elements were considered in Figure 2 for the

Table 1. Grid refinement check at $\operatorname{Pr}=0.71$ and $R i=1$.

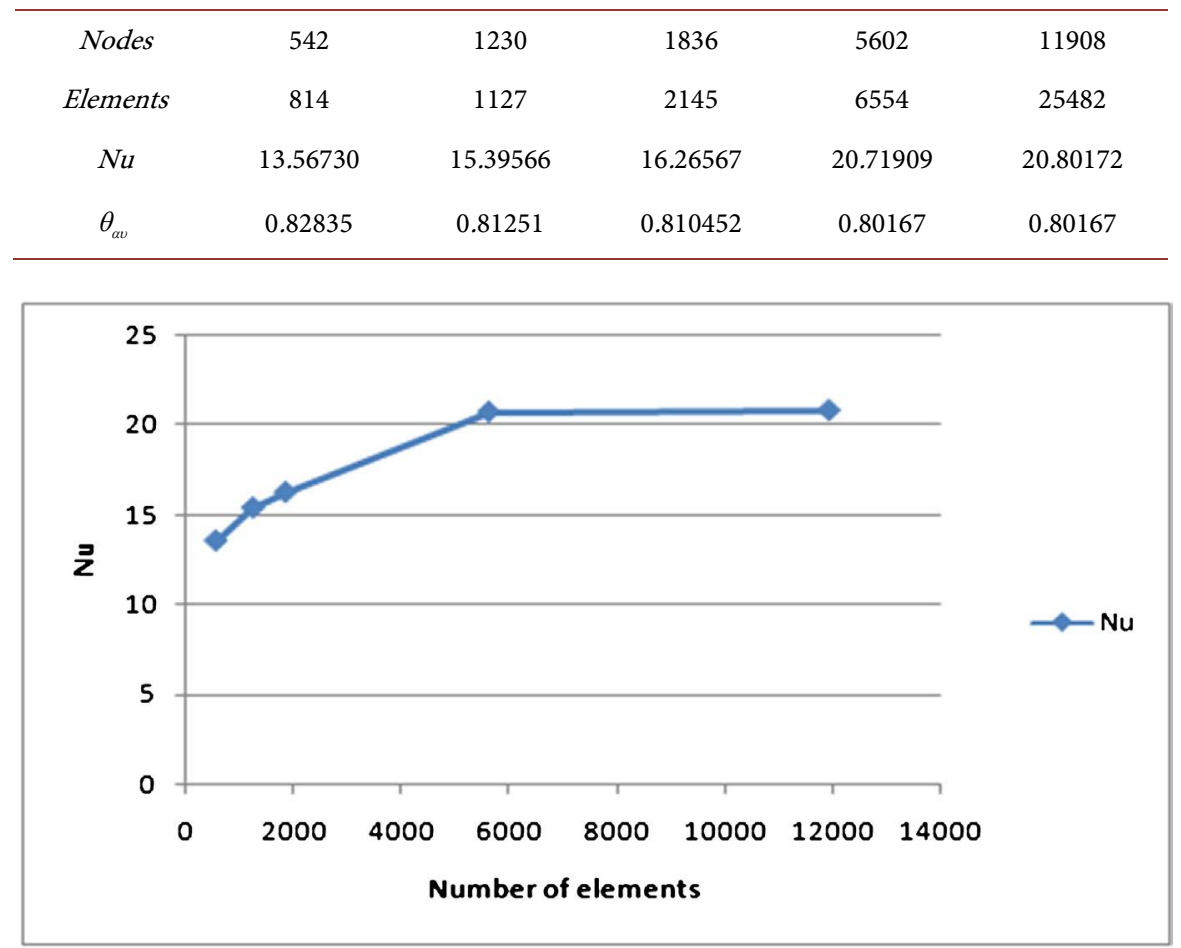

Figure 2. Grid independency study on average Nusselt number in the cavity while $\operatorname{Pr}=0.71$ and $R i=1$. 
grid refinement tests. From these values, 11,908 nodes, 25,482 elements can be chosen throughout the simulation to optimize the relation between the accuracy required.

\section{Result and Discussion}

In this section, some representative results are presented to illustrate the effects of various controlling parameters on the fluid flow. These controlling parameters include Richardson number ranging from 0.01 to 10 and the Hartmann number varying from 0 to 50 with fixed Reynolds number $R e=100$ and Prandtl number $\operatorname{Pr}=0.71$. The results are presented in terms of streamlines, isotherms, local Nusselt number, velocity profiles and dimensionless temperature profile along the vertical wall.

Streamlines for different values of Richardson number $R i=0.01-10$ while $H a=0$ and $\operatorname{Pr}=0.71$ are presented in Figure 3. At $R i=0.01$ and in the absence of magnetic field ( $H a=0$ ), two circular cells are formed in lower side of square heated block of the cavity shown in Figure 3(a).

As $R i$ increases up to 1 , the effect of mixed convection increases and both the primary and secondary circulation do increase. It is observed that moving lids become very strong. For higher Richardson number one circulation cell is formed at upper right corner of the cavity and also the increase of flow strength is shown in Figure 3(d).
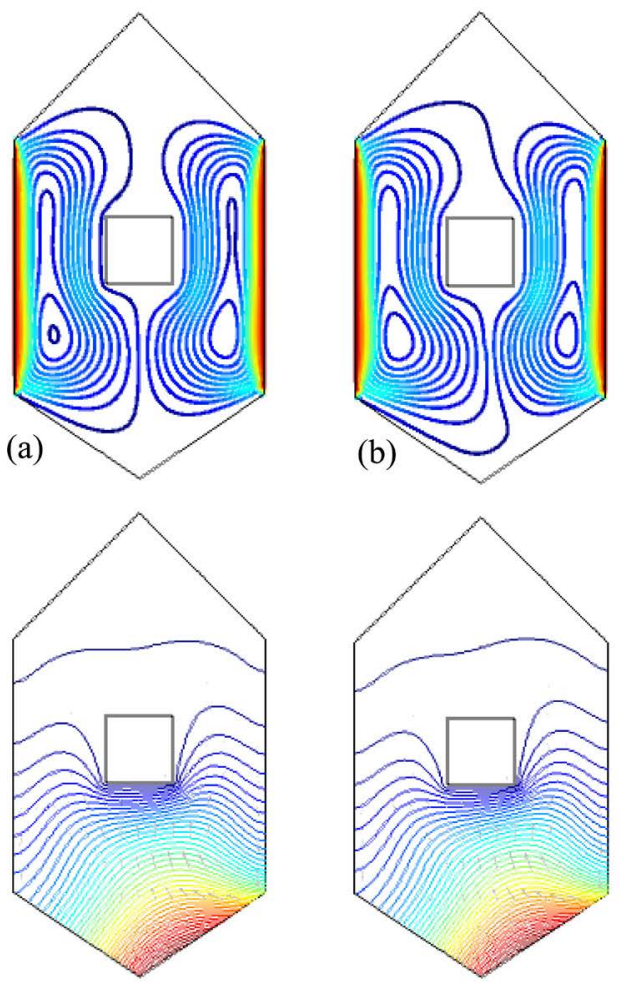

(e)

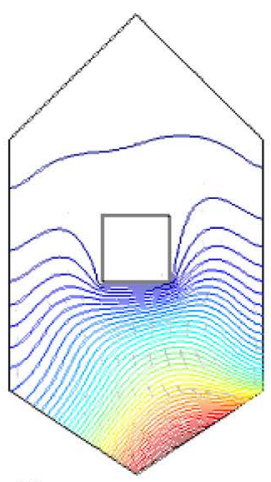

(f)

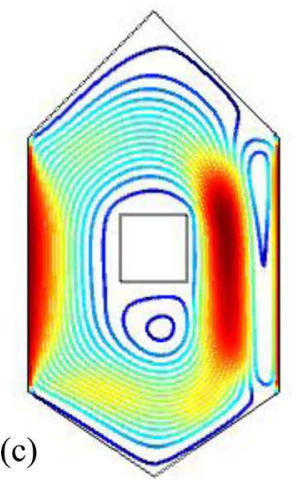

(d)
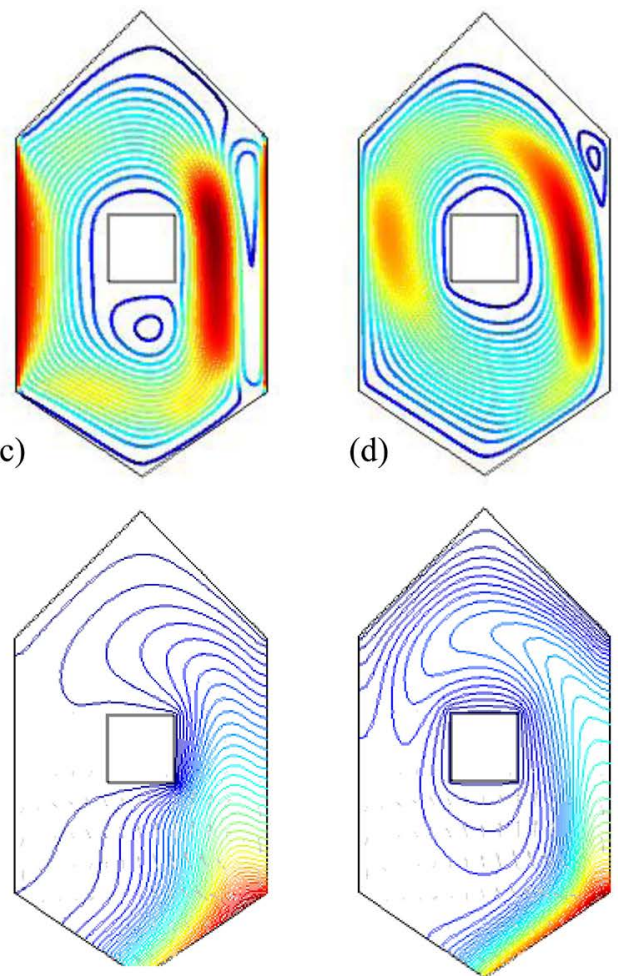

(g)

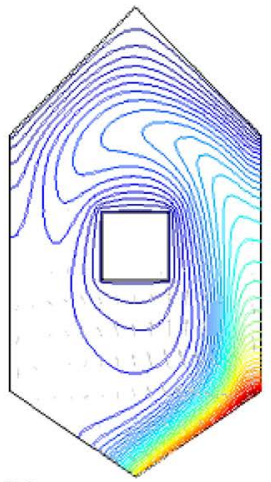

(h)

Figure 3. Streamlines and isotherms for different values of Richardson number $R i=0.01-10$ while $H a=0$ and $P r=0.71$. 
Conduction dominant heat transfer is observed from the isotherms in Figures 3(e)-(h) at $H a=0$ and $P r=0.71$ respectively. As seen from the figures, isotherms concentrate near the bottom wall and isotherm lines are more bending which means increasing heat transfer throw convection. For higher Richardson number, the isotherm lines are bending near right side wall that means increasing heat transfer throw convection are shown in Figure 3(h).

Variation of streamlines and isotherms for different values of Richardson number $R i=0.01-10$ while $H a=50$ and $P r=0.71$ are shown in Figure 4. It can be found that the strength of buoyancy inside the cavity is significant and more fluid rises from two sided lid-driven cavity. As Ri increases, the strength of buoyancy increases and circulation cells inside the cavity appear in Figures 4(a)-(d). At $H a=50$ and $R i=0.01-10$, the isotherms illustrate a pure conduction heat transfer. In Figures $4(\mathrm{e})$-(h), the isotherm lines bending at middle of the hexagonal cavity. While $R i$ increases, the strength of the buoyancy increases and isotherm lines bend at the lower corner heater with increase of heat transfer.

The local Nusselt Number along the vertical wall for different Richardson numbers $R i=0.01-10$ with $H a=0$ and $P r=0.71$ of the cavity is shown in Figure 5(a). Maximum and minimum shape curves are obtained here. For $x<0.6$ maximum shape curve are found and $x>0.6$ the minimum shape curve are found and absolute value of local Nusselt number (that is heat transfer rate)

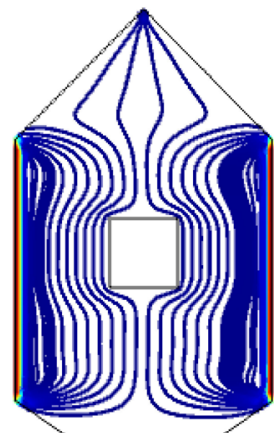

(a)

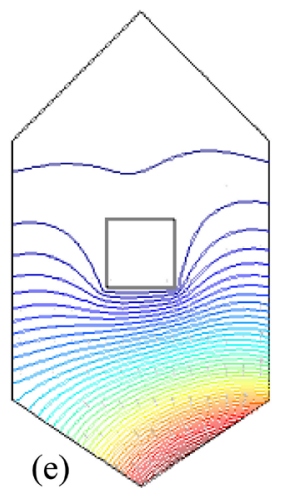

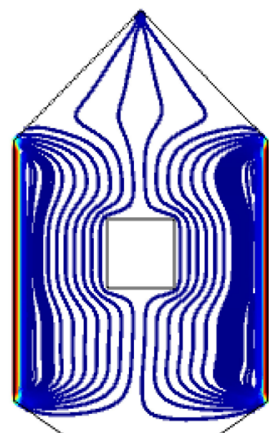

(b)

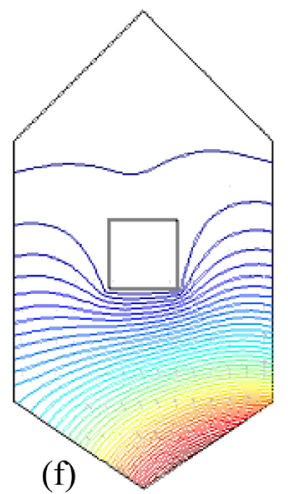

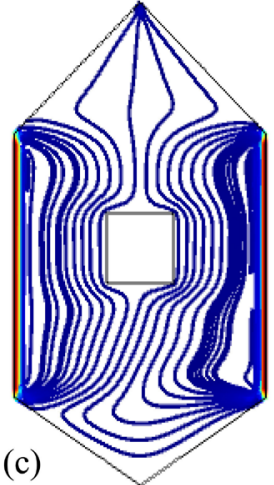

(c)

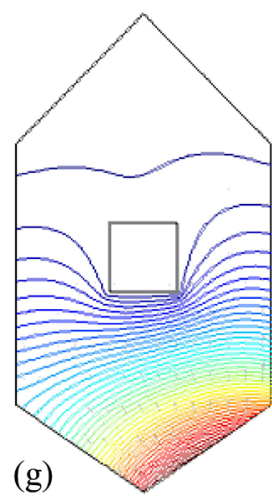

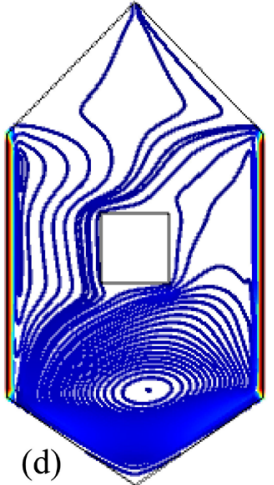

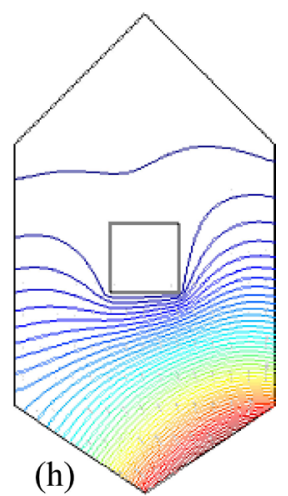

Figure 4. Streamlines and isotherms for different values of Richardson number $R i=0.01-10$ while $H a=50$ and $\operatorname{Pr}=0.71$. 


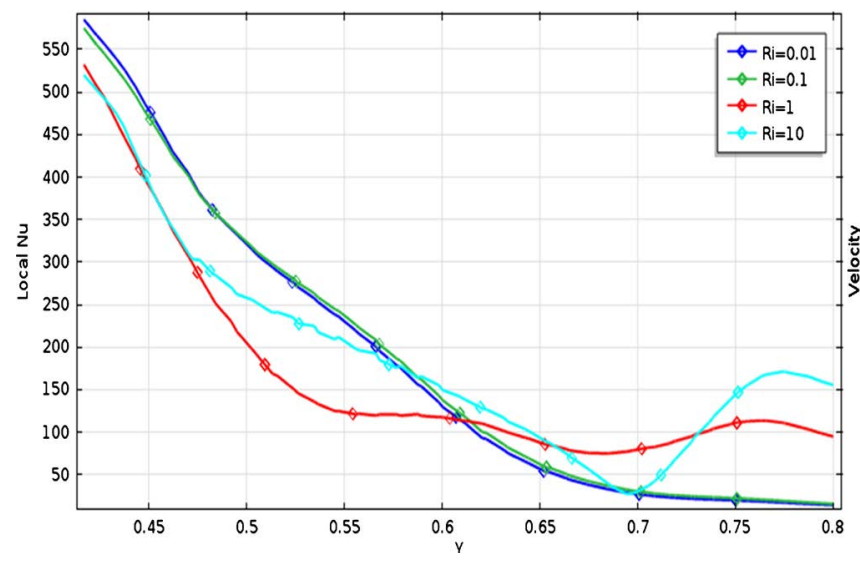

(a)

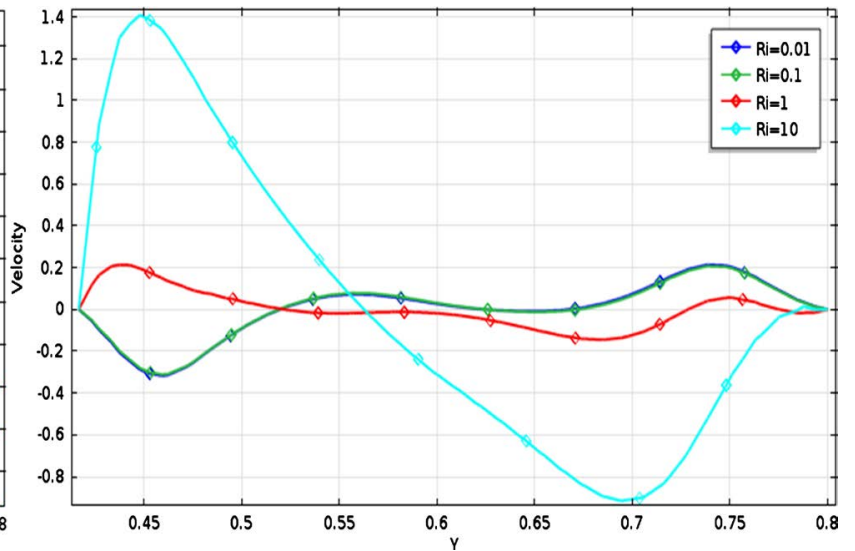

(b)

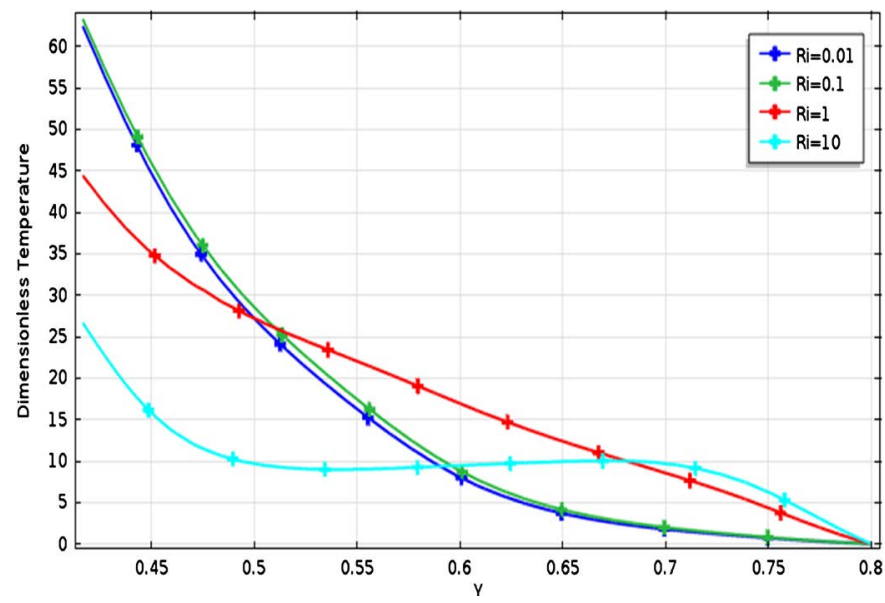

(c)

Figure 5. Variation of (a) Local Nusselt number, (b) Velocity and (c) Dimensionless Temperature for different values of Richardson number $R i=0.01-10$ while $H a=0$ and $P r=0.71$.

is increased with the increase of Richardson number.

Variation of the vertical velocity component along the horizontal line of the cavity with different Richardson numbers and $H a=0$ is shown in Figure 5(b). From the figure it is evident that for lower values of Richardson number the value of the velocity has smaller change but for the higher values of Richardson number the value of the velocity has larger change. Figure 5(c), presents the dimensionless temperature profiles along the horizontal wall for different values of Richardson number with $H a=0$ and $P r=0.71$. Maximum and minimum temperature curves are obtained here. For the lower values of Richardson number the temperature increases and for the higher values of Richardson number the temperature decreases.

The local Nusselt numbers along vertical wall for different values of Richardson number with $H a=50$ and $P r=0.71$ of the cavity are shown in Figure 6(a). It can be seen from the Figure 6(a), in whole portion of the cavity the Local Nusselt number decreases with the increase of Richardson number and does not change significantly with increase of the Richardson number. 


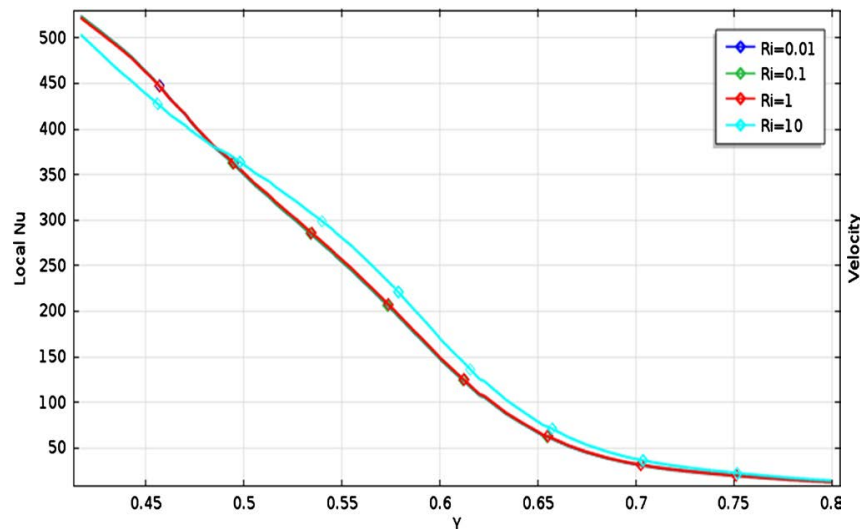

(a)

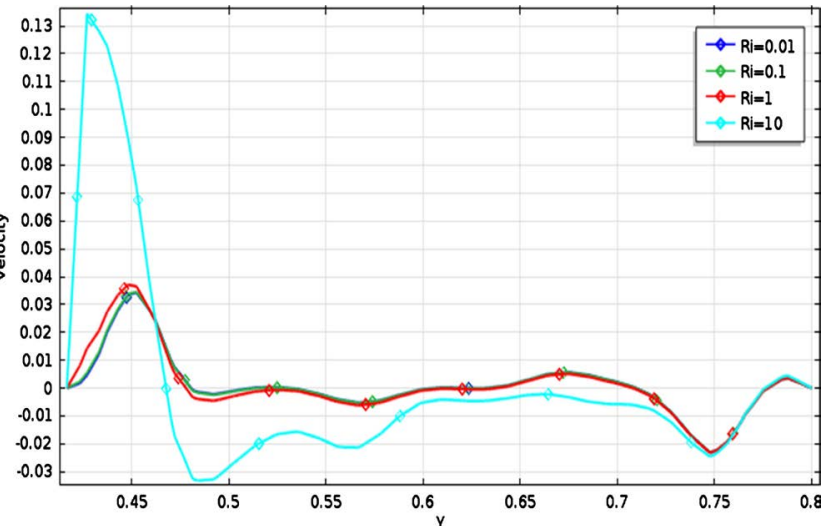

(b)

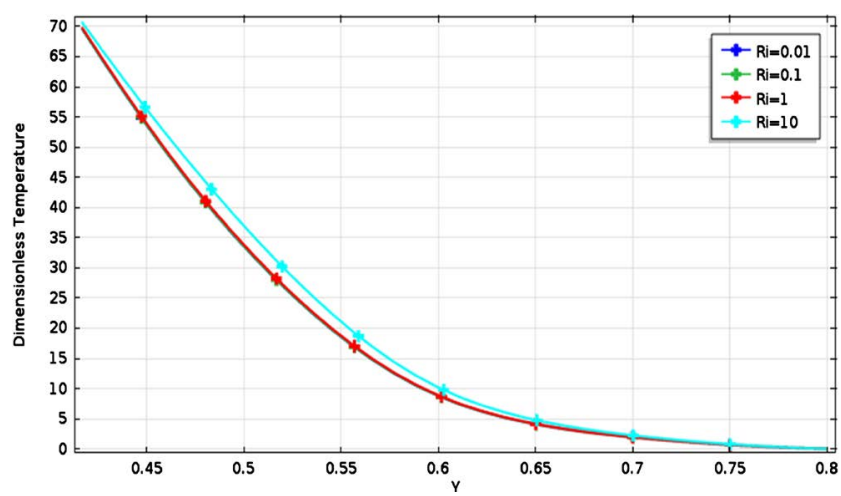

(c)

Figure 6. Variation of (a) Local Nusselt number, (b) Velocity and (c) Dimensionless Temperature for different values of Richardson number $R i=0.01-10$ when $H a=50$ and $P r=0.71$.

Variations of the vertical velocity component along the horizontal line of the cavity with the Richardson number and for $H a=50$ are shown in Figure 6(b). It can be seen from the Figure, in whole portion of the cavity the velocity decreases with increase of Richardson number and finally the rate of heat transfer decreases.

Figure $6(\mathrm{c})$ presents the dimensionless temperature profile along the horizontal wall for different values of Richardson number with $H a=50$ and $P r=0.71$. At $H a=50$ the thickness of the boundary layer decreases. For the lower Richardson number temperature has more significant changes but for higher Richardson number less significant.

Streamlines for $G r=10^{4}, R e=100$ and $P r=0.71$ are presented in Figures 7(a)-(d) respectively. These figures show the effects of Hartmann number on flow field and temperature distribution. From the streamlines it is found that with the increase of Hartmann number (increase of the strength of the magnetic field), flow strength decreases. At $H a=0$ two circular shapes are formed at the below of the heated block of the cavity shown in Figure 7(a). For higher Hartmann number one circular shape formed at the below of the heated block inside the cavity and also the decreases of the flow strength are shown in Figures 7 (b) -(d). 


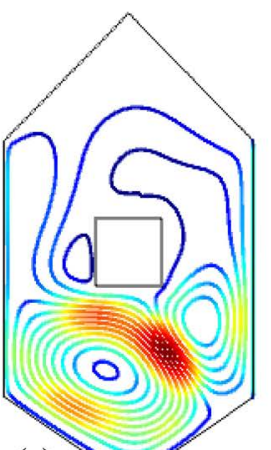

(a)

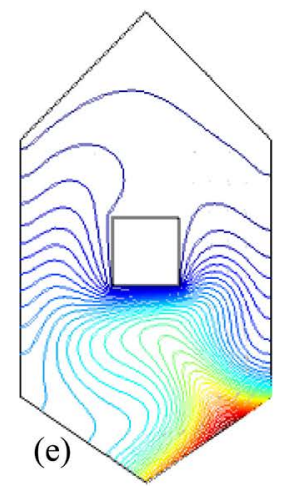

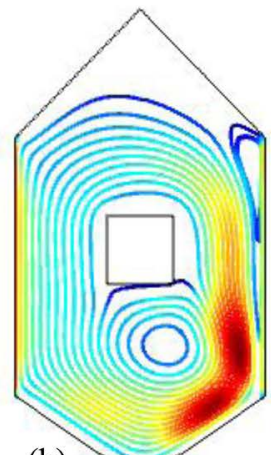

(b)

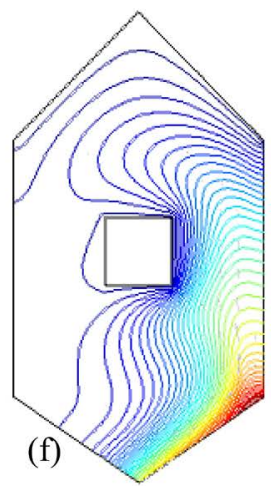

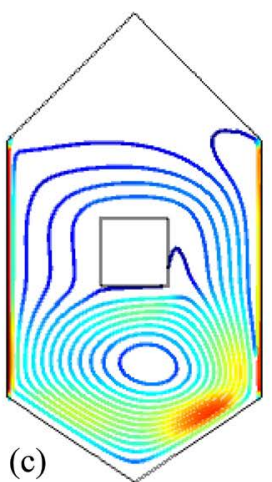
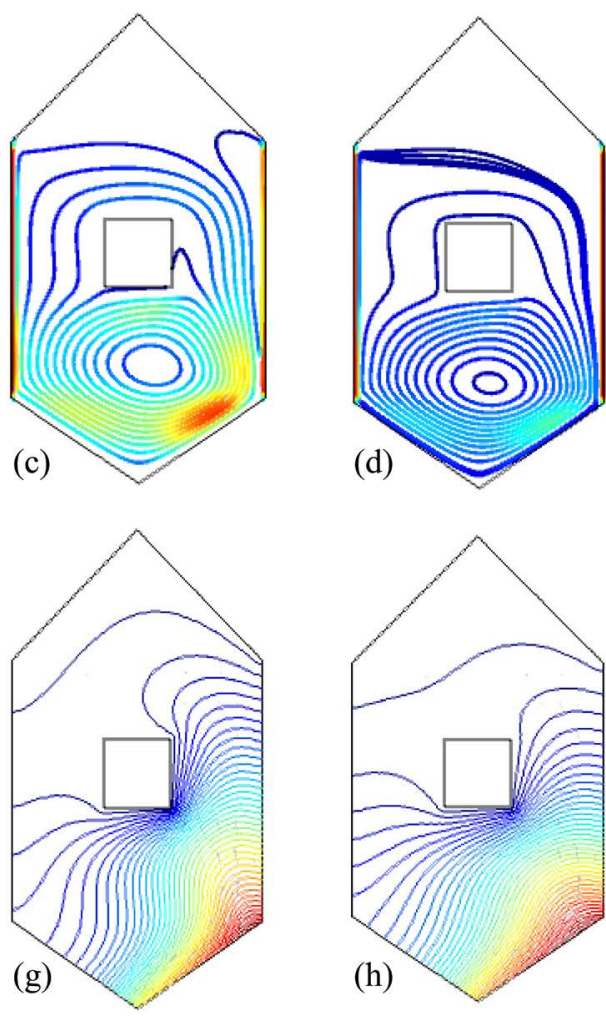

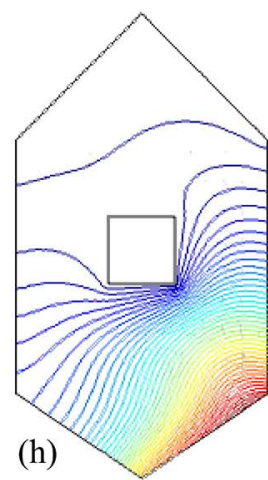

Figure 7. Streamlines and isotherms for different values of Hartmann number $H a=0,10,20,30$ when $G r=10^{4}, \operatorname{Re}=100$ and $\operatorname{Pr}=0.71$.

Conduction dominant heat transfer is observed from the isotherms in Figures 7(e)-(h) at $G r=10^{4}, R e=100$ and $\operatorname{Pr}=0.71$. At $H a=0$ the isotherm lines near the bottom wall and isotherm lines are more bending which means increasing heat transfer throw convection. Formation of the thermal boundary layers can be found that it increases from the isotherms for $H a=0-30$. The isotherms are almost parallel to lid-driven wall and they show a linear increase along the corner heater.

The local Nusselt Number along vertical wall for different values of Hartmann number with $G r=10^{4}, R e=100, P r=0.71$ and $R i=1$ of the cavity are shown in Figure 8(a). It can be seen from the figure, in whole portion of the cavity the local Nusselt number decreases with increase of the Hartmann number.

Variation of the vertical velocity component along the horizontal line of the cavity with $G r=10^{4}, R e=100, \operatorname{Pr}=0.71, R i=1$ and different values of the Hartmann number are shown in Figure 8(b). It can be seen from the Figure that the absolute value of maximum and minimum value of velocity increases with the increase of the Hartmann number (increase of the buoyancy force).

Figure $8(\mathrm{c})$ presents the dimensionless temperature profile along the horizontal wall for different values of Hartmann number with $G r=10^{4}, R e=100$, $P r=0.71$ and $R i=1$. When the values of Hartmann number increases, then the dimensionless temperature decreases. 


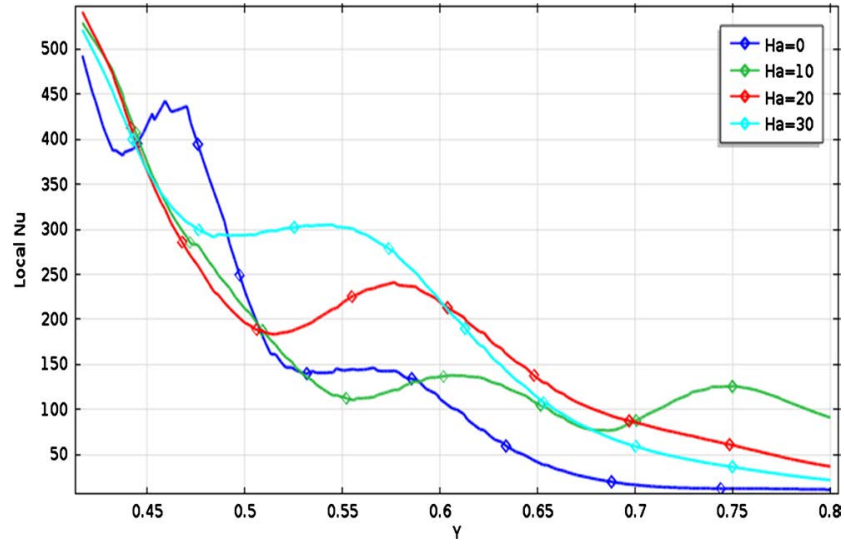

(a)

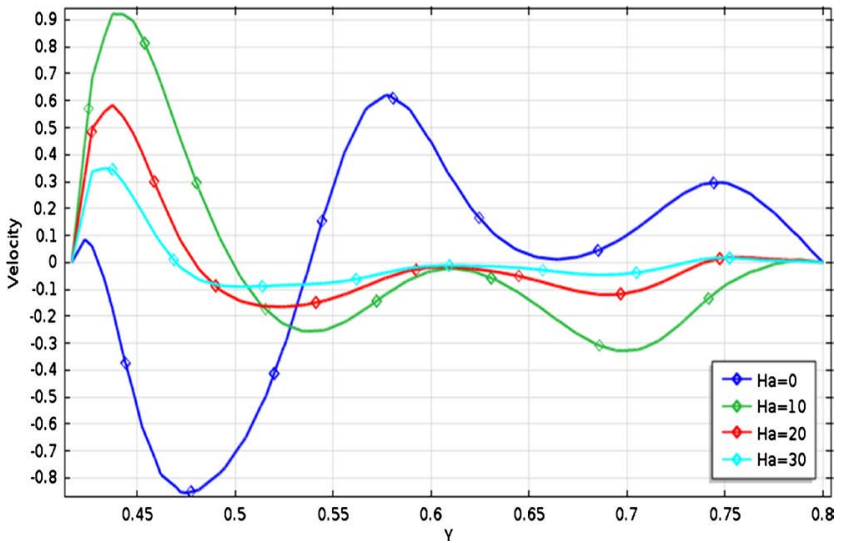

(b)

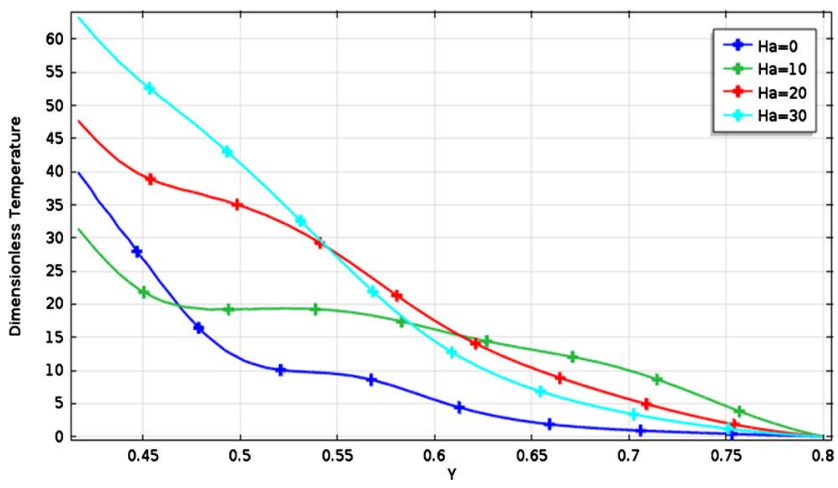

(c)

Figure 8. Variation of (a) Local Nusselt number; (b) Velocity and (c) Dimensionless Temperature for different values of Hartmann number $H a=0,10,20,30$ when $G r=10^{4}, \operatorname{Re}=100$ and $\operatorname{Pr}=0.71$.

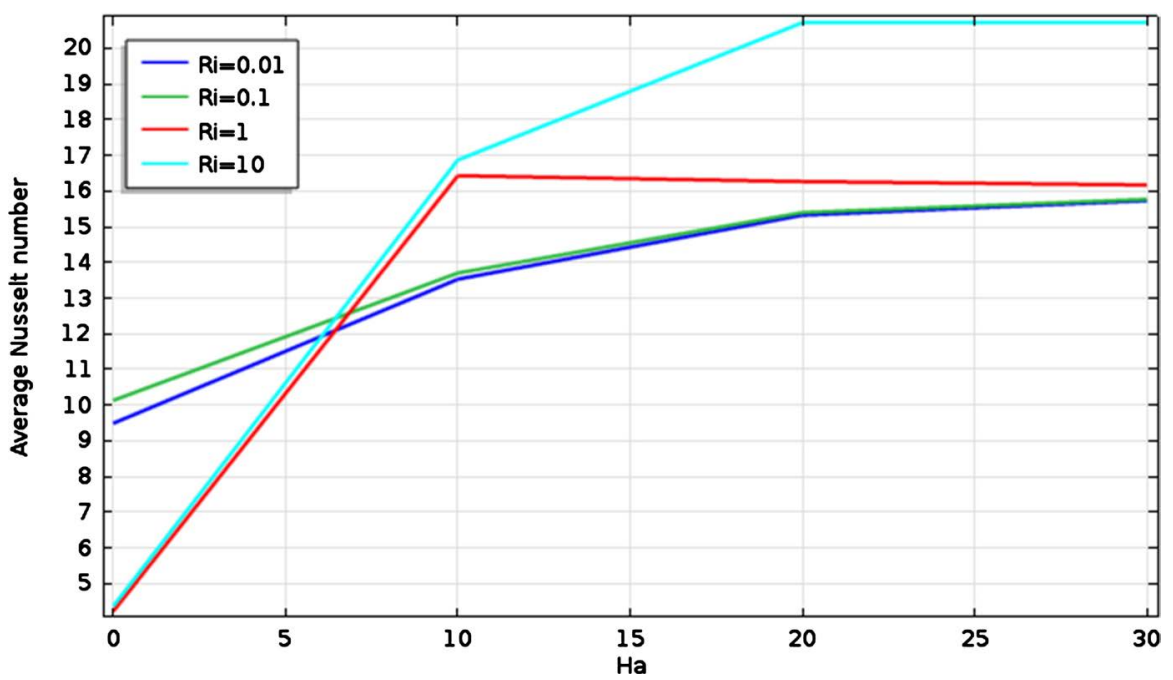

Figure 9. Variation of average Nusselt number versus Hartmann number for different Richardson number $R i=0.01,0.1,1,10$ when $G r=10^{4}, R e=100, H a=50$ and $\operatorname{Pr}=0.71$.

The influence of Richardson number on average Nusselt number versus different Hartmann numbers for vertical wall is displayed in Figure 9 at $\mathrm{Gr}=10^{4}$, $R e=100, H a=50$ and $P r=0.71$. It is clearly observed that adding Richard- 
son number inside the hexagonal enclosure cause an increase in the average Nusselt number. Also rate of increment on heat transfer clearly depends on the value of Richardson number.

\section{Conclusions}

Numerical study on mixed convection in a lid-driven hexagonal cavity with corner heater has been performed. Results have been presented in terms of streamlines, isotherms, local Nusselt number, velocity profile and dimensionless temperature. The results are obtained for a wide range of pertinent dimensionless groups such as Richardson number and Hartmann numbers. In view of the obtained results, the following findings are précised:

1) The flow characteristics and heat transfer mechanism inside the hexagonal cavity are strongly dependent on the Richardson number.

2) The significant suppression of the convective current in the enclosure is due to increase of Hartmann numbers.

3) For all cases considered, two counter rotating eddies were formed inside the cavity regardless the Richardson number and the Hartmann numbers. The temperature distribution and the flow characteristics inside the cavity strongly depend on both the strength of the magnetic field and the Richardson number.

4) The large values of Richardson number lead to increase the lid-driven effect whereas the small values of Richardson number lead to increase effect of presence of the heat source on the flow and heat characteristics.

\section{Conflicts of Interest}

The authors declare no conflicts of interest regarding the publication of this paper.

\section{References}

[1] Al-Amiri, A.M., Khanafer, K.M. and Pop, I. (2007) Numerical Simulation of Combined Thermal and Mass Transport in a Square Lid-Driven Cavity. International Journal of Thermal Sciences, 46, 662-671. https://doi.org/10.1016/j.ijthermalsci.2006.10.003

[2] Sharif, M.A.R. (2007) Laminar Mixed Convection in Shallow Inclined Driven Cavities with Hot Moving Lid on Top and Cooled from Bottom. Applied Thermal Engineering, 27, 1036-1042. https://doi.org/10.1016/j.applthermaleng.2006.07.035

[3] Chen, C.-L. and Cheng, C.-H. (2009) Numerical Simulation of Periodic Mixed Convective Heat Transfer in a Rectangular Cavity with a Vibrating Lid. Applied Thermal Engineering, 29, 2855-2862.

https://doi.org/10.1016/j.applthermaleng.2009.02.009

[4] Khanafer, K.M., Al-Amiri, A.M. and Pop, I. (2007) Numerical Simulation of Unsteady Mixed Convection in a Driven Cavity Using an Externally Excited Sliding Lid. European Journal of Mechanics, 26, 669-687. https://doi.org/10.1016/j.euromechflu.2006.06.006

[5] Teamah, M.A. and El-Maghlany, W.M. (2010) Numerical Simulation of Double-Diffusive Mixed Convective Flow in Rectangular Enclosure with Insulated 
Moving Lid. International Journal of Thermal Sciences, 49, 1625-1638. https://doi.org/10.1016/j.ijthermalsci.2010.04.023

[6] Saha, L.K., Uddin, K.M.S. and Taher, M.A. (2015) Effect of Internal Heat Generation or Absorption on MHD Mixed Convection Flow in a Lid Driven Cavity. American Journal of Applied Mathematics, 3, 20-29. https://doi.org/10.11648/j.ajam.s.2015030101.13

[7] Dawood, M.M.K. and Teamah, M.A. (2012) Hydro-Magnetic Mixed Convection Double Diffusive in a Lid Driven Square Cavity. European Journal of Scientific Research, 85, 336-355.

[8] Hussein, N.A. (2013) Study of Mixed Convection in Square Lid-Driven with Eccentric Circular Body. Journal of Babylon University/Engineering Sciences, 21, 616-634.

[9] Nasrin, R. (2011) Aspect Ratio Effect of Vertical Lid Driven Chamber Having a Centered Conducting Solid on Mixed Magneto Convection. Journal of Scientific Research, 3, 501-513. https://doi.org/10.3329/jsr.v3i3.7433

[10] Billah, M.M., Rahman, M.M., Sharif, U.M., Rahim, N.A., Saidur, R. and Hasanuzzaman, M. (2011) Numerical Analysis of Fluid Flow Due to Mixed Convection in a Lid-Driven Cavity Having a Heated Circular Hollow Cylinder. International Communications in Heat and Mass Transfer, 38, 1093-1103. https://doi.org/10.1016/j.icheatmasstransfer.2011.05.018

[11] Munshi, M.J.H., Alim, M.A., Bhuiyan, A.H. and Ali, M. (2017) Hydrodynamic Mixed Convection in a Lid-Driven Square Cavity Including Elliptic Shape Heated Block with Corner Heater. Procedia Engineering, 194, 442-449.

https://doi.org/10.1016/j.proeng.2017.08.169

[12] Sivasankaran, S., Malleswaran, A. and Lee, J. (2011) Hydro-Magnetic Combined Convection in a Lid-Driven Cavity with Sinusoidal Boundary Conditions on Both Sidewalls. International Journal of Heat and Mass Transfer, 54, 512-525. https://doi.org/10.1016/j.ijheatmasstransfer.2010.09.018 


\section{Nomenclatures}

\begin{tabular}{llll}
\hline$g$ & Gravitational attraction & $X, Y$ & Non-dimensional Cartesian coordinates \\
$\mathrm{Nu}$ & Nusselt number & $U, V$ & Non-dimensional velocity components \\
$P$ & Dimensional Pressure & \multicolumn{2}{c}{ Greek Symbols } \\
$p$ & Pressure & $\alpha$ & Thermal diffusivity \\
$P r$ & Prandtl number & $\beta$ & Volumetric coefficient of thermal expansion \\
$\mathrm{Ha}$ & Hartmann number & $v$ & Kinematic viscosity of the fluid \\
$R e$ & Reynolds number & $\theta$ & Non-dimensional temperature \\
$R i$ & Richardson number & $\rho$ & Density of the fluid \\
$G r$ & Grashof number & Subscripts \\
$X, y$ & Cartesian coordinates & $c$ & Cold wall \\
$u, V$ & Velocity component & $h$ & Hot wall \\
$T$ & Temperature & \multicolumn{2}{c}{}
\end{tabular}

